\title{
Microsatellite loci discovery from next-generation sequencing data and loci characterization in the epizoic barnacle Chelonibia testudinaria (Linnaeus, 1798)
}

Christine Ewers-Saucedo, John D Zardus, John P Wares

Microsatellite markers remain an important tool for ecological and evolutionary research, but are unavailable for many non-model organisms. One such organism with rare ecological and evolutionary features is the epizoic barnacle Chelonibia testudinaria (Linnaeus, 1758). Chelonibia testudinaria appears to be a host generalist, and has an unusual sexual system, androdioecy. Genetic studies on host specificity and mating behavior are impeded by the lack of fine-scale, highly variable markers, such as microsatellite markers. In the present study, we discovered thousands of new microsatellite loci from next-generation sequencing data, and characterized 12 loci thoroughly. We conclude that 11 of these loci will be useful markers in future ecological and evolutionary studies on $C$. testudinaria. 
1 Microsatellite loci discovery from next-generation sequencing data and loci

2 characterization in the epizoic barnacle Chelonibia testudinaria (Linnaeus, 3 1798)

4

5 Christine Ewers-Saucedo ${ }^{1,2}$, John D. Zardus ${ }^{3}$, John P. Wares ${ }^{1,4}$

6

$7{ }^{1}$ Department of Genetics, University of Georgia, Athens, GA, USA

82 Evolution and Ecology, University of California, Davis, CA, USA

$9{ }^{3}$ Department of Biology, The Citadel, Charleston, SC, USA

$10{ }^{4}$ Odum School of Ecology, University of Georgia, Athens, GA, USA

11

12 Corresponding author:

13 Christine Ewers-Saucedo

14 Storer Hall, Davis, CA 95616, USA

15 Email address: ewers.christine@gmail.com

16 


\section{Abstract}

18 Microsatellite markers remain an important tool for ecological and evolutionary research, but are

19 unavailable for many non-model organisms. One such organism with rare ecological and

20 evolutionary features is the epizoic barnacle Chelonibia testudinaria (Linnaeus, 1758).

21 Chelonibia testudinaria appears to be a host generalist, and has an unusual sexual system,

22 androdioecy. Genetic studies on host specificity and mating behavior are impeded by the lack of

23 fine-scale, highly variable markers, such as microsatellite markers. In the present study, we

24 discovered thousands of new microsatellite loci from next-generation sequencing data, and

25 characterized 12 loci thoroughly. We conclude that 11 of these loci will be useful markers in

26 future ecological and evolutionary studies on C. testudinaria. 


\section{Introduction}

Microsatellite loci are valuable tools in ecological and evolutionary studies (e.g. Jarne and Lagoda, 1996; Vignal et al. 2002; Selkoe and Toonen, 2006). Next-generation sequencing approaches have revolutionized microsatellite loci development, allowing the rapid discovery of thousands of potential microsatellite loci in the genome of non-model organisms (Castoe et al., 2012). However, a thorough characterization of potential microsatellite loci remains laborintensive. It is nonetheless necessary if we want to use these markers successfully in evolutionary and ecological studies.

A non-model organism for which genetic and genomic resources are lacking is the epizoic barnacle Chelonibia testudinaria (Linnaeus, 1758). Chelonibia testudinaria uses diverse marine animals as substratum, such as sea turtles, manatees, swimming crabs, and horseshoe crabs. Host-specific morphotypes were previously described as distinct species (Darwin, 1854; Hayashi, 2013). Recent molecular analyses indicate that C. testudinaria is a host generalist, and C. patula (Ranzani, 1818) and C. manati (Gruvel, 1903) are now considered synonyms of $C$. testudinaria (Cheang et al., 2013; Zardus et al., 2014). However, a fine-scale genetic assessment of host-specificity based on highly polymorphic nuclear markers, such as microsatellite markers, is still lacking.

While host species do not seem to provide barriers to gene flow, biogeography does:

Three major genetic lineages are restricted to the Indo-West Pacific, Tropical Eastern Pacific and Atlantic Ocean, respectively (Rawson et al., 2003, Cheang et al., 2013; Zardus et al., 2014).

51 (Zardus et al., 2014).

All lineages of C. testudinaria exhibit a rare sexual system: androdioecy. Androdioecy is 
53 characterized by the co-existence of hermaphrodites and males in the same reproductive

54 population. Understanding mating success and mating patterns of both sexes would greatly

55 advance our understanding of this rare sexual system. This would be most easily achieved with

56 genetic parentage assignment - but its prerequisite, highly variable genetic markers, are not

57 available.

58 In order to overcome these shortcomings, we used next-generation sequencing to discover

59 microsatellite markers for C. testudinaria. We characterized 12 promising markers in

60 populations from the Atlantic coast of the United States and from the Northeastern coast of

61 Australia.

62

63

64 Material \& Methods

65

66 Specimen collections

67 Specimen collections of the Atlantic lineage took place on Nannygoat Beach, Sapelo

68 Island, GA, USA $\left(31.48^{\circ} \mathrm{N}, 81.24^{\circ} \mathrm{W}\right)$ between 2012 and 2014 under the collection permit of

69 the University of Georgia Marine Institute, and sanctioned by the Georgia DNR Wildlife

70 Services. We chose to collect from the horseshoe crab Limulus polyphemus (Linnaeus, 1758)

71 because it is relatively abundant and easy to sample: each spring and early summer, horseshoe

72 crabs crawl onto beaches to mate and lay their eggs. During this process, we removed one

73 individual of C. testudinaria per host individual with a sharp knife directly on the beach, and

74 preserved it in $95 \%$ EtOH immediately after collection. We collected specimens of the Indo-

75 West Pacific lineage in the vicinity of Townsville, Queensland, Australia $\left(23^{\circ} \mathrm{S}, 143^{\circ} \mathrm{E}\right)$, in 
76 September 2012 from green turtles (Chelonia mydas Brongniart, 1800). Working with officials

77 from the Queensland Department of Environment and Heritage Protection, turtles were captured

78 in-water for routine tagging and release. During capture barnacles were collected and

79 immediately preserved in $95 \% \mathrm{EtOH}$.

80

81 Microsatellite loci discovery

We extracted genomic DNA from the feeding appendage of a single large hermaphroditic

C. testudinaria collected from a horseshoe crab with Gentra Puregene Tissue Kit (Qiagen), and

measured DNA concentration with a Qubit 2.0 Fluorometer (Life Technologies). Genomic DNA

was fragmented into approximately 700bp lengths (insert size) and shotgun-sequenced on an

Illumina MiSeq sequencer (PE250). We quality-checked paired-end reads with FastQC

87 (Andrews, 2015). The software FASTQMCF was used to trim adapters, cut low quality ends and remove low quality reads and their mate-pair read (Aronesty, 2011).

We executed the perl script PALFINDER to identify short sequence repeat regions (Castoe et al., 2012). The script also calls PRIMER3, version 2.0.0, to identify potential primer pairs that

91 span the repeat region (Rozen \& Skaletsy, 2000). The minimum number of repeat units was chosen as in Castoe et al. (2012). A repeat unit, also called kmer, is defined as the length of the short sequence repeat. For example, a dimer would be a repeat of two base pairs (e.g. GC), and a tetramer would be a repeat of four basepairs (e.g. AGGT). PRIMER3 parameters were the default

95 values. The search resulted in a large number of potentially amplifiable loci (PALs), repeat

96 regions for which primers were identified. We filtered the results by removing all PALs which

97 occurred less than two times and more than the estimated genome coverage in the genomic reads

98 based on the following reasoning: If the number of primer occurrences is low, the primer 
99 sequence may contain sequencing error. If the number of occurrences is higher than the expected

100 genome coverage, the primer region may occur more than once in the genome, leading to

101 amplification of multiple loci (genomic regions). Neither of these outcomes is desirable because

102 a good marker occurs only once in the genome, and has a primer sequence that matches the

103 genomic sequence well. We estimated genome coverage by mapping our genomic reads to 52

104 nuclear single-copy gene fragments available from the acorn barnacle Semibalanus balanoides

105 (Regier et al., 2010) with the Geneious version 6.0.3 Read Mapper, using the default settings

106 (Kearse et al., 2012), calculated the median coverage for each of the 52 gene fragments, and the

107 grand median of all genes. R scripts for screening PALFINDER output as well as calculating

108 genome coverage are available as supplementary information.

109 Of the filtered PALs, we chose 48 PALs for trial amplification, which differed in kmer

110 length, kmer motif (e.g. AG vs TG) and fragment size. We extracted and amplified DNA of 16

111 C. testudinaria individuals for trials. DNA was extracted from feeding appendages of barnacles

112 with the Chelex method (Walsh et al., 1991). Trials used the method of Schuelke (2000) to

113 amplify fragments and simultaneously tag forward primers with a fluorescent dye. Loci that

114 amplified and scored consistently in all individuals were fluorescently labeled with 6-FAM,

115 NED or HEX (Applied Biosystems, Custom Oligo Synthesis Center), and used on a larger

116 number of individuals to characterize the microsatellite loci.

117

118 Microsatellite loci amplification

119 Genomic DNA was extracted from feeding appendages of barnacles with the Chelex

120 method (Walsh et al., 1991). PCR amplifications were performed in 20ul volumes containing

121 final concentrations of $1 x$ PCR buffer (Bioline), 5\% bovine serum albumin $10 \mathrm{mg} / \mathrm{mL}$ (Sigma), 
$122200 \mathrm{mM}$ each dNTP, $2 \mathrm{nM} \mathrm{MgCl}, 0.5 \mathrm{mM}$ each primer, 0.5 units of Promega GoTaq DNA

123 Polymerase, and $1 \mu 1$ template DNA. PCR conditions were as follows: 4 min initial denaturation,

124 followed by 40 cycles with $45 \mathrm{sec}$ denaturing at $94^{\circ} \mathrm{C}, 60 \mathrm{sec}$ annealing at $55^{\circ} \mathrm{C}, 60 \mathrm{sec}$ extension

125 at $72^{\circ} \mathrm{C}$ and a final extension time of $10 \mathrm{~min}$. The PCR were carried out in a MJ Research PCR

126 Engine. HiDi and ROX500 size standard were added to each sample, and fragment length

127 analysis was carried out at the Georgia Genomics Facility on an ABI 3730xl. Peaks were called

128 and binned with the microsatellite plugin of Geneious version 8.1 (Kearse et al., 2012).

130 Microsatellite loci characterization

131 We first characterized the microsatellite loci on 42 individuals from the Atlantic

132 population. We inspected peak calls for fragment size consistency, using the R package

133 MsatAlLele (Alberto, 2009). MsatAlLELE plots peak calls of a locus in histogram form,

134 facilitating visual binning of alleles. If bins could not be clearly assigned, the locus was excluded

135 from the subsequent analysis. We tested whether loci were in Hardy-Weinberg Equilibrium

136 (HWE) by using 1999 Monte Carlo permutations, as implemented by the function HW.TEST in the

137 R package PEGAS (Paradis, 2010). Significance values were adjusted for multiple comparisons

138 based on the method of Holm (1979). We recorded the number of alleles, range of fragment

139 sizes, and allelic richness of each locus. The frequency of null alleles was computed based on the

140 method of Brookfield (1996). Genotyping error rates were calculated by repeating genotyping

141 for all individuals. After characterizing the loci in the Atlantic population, we amplified the loci

142 for 24 individuals from Queensland, Australia, to assess if the loci could be used in cross-lineage

143 analysis. Characterization on individuals of the Indo-West Pacific population were largely the

144 same as for the Atlantic population, but we did not assess genotyping error rates. A R script 
145 detailing these analyses is available as supplementary information.

\section{Results}

149

150 Microsatellite marker development

The MiSeq run generated 15,324,079 paired-end reads (35-251 bp long) with $81.05 \%>$

152 Q30. Raw reads are available in NCBI's short read archive (Study accession: SRP069253, run

153 accession: SRR3144544). After quality control, 13,498,280 paired-end reads (19-251 bp long)

154 remained, for a total of $6.2 \mathrm{~Gb}$. The median genome coverage was $8 \mathrm{x}(\min =3$, $\max =24)$ for 52

155 nuclear single-copy gene fragments. The PALFINDER script detected 629,990 microsatellite repeat

156 regions, of which 29,627 (5.38\%) were potentially amplifiable loci (PALs) with forward and

157 reverse primer. A summary of detected microsatellite repeat regions is available as

158 supplementary information. A list of all detected microsatellite regions (with primer sequences)

159 is available on figshare (www.figshare.com; DOI: 10.6084/m9.figshare.2070070). After

160 removing PALs with more than eight or less than two occurrences of either forward or reverse

161 primer in the sequence read data, 17,265 PALs remained. We chose 48 perfect repeat loci for

162 trial amplification, which differed in kmer length and repeat motif, but were otherwise chosen at

163 random. Of those 48 loci, 12 loci amplified and scored consistently throughout the trials, and

164 were tagged with fluorescently labeled dye (Table 1).

165

166 Microsatellite marker characterization

167 We genotyped 42 individuals successfully at more than half of the 12 consistently scoring 
168 loci. Visual inspection of peak call histograms revealed that peak calls of Ctest 2 did not have

169 clearly defined bins, and were excluded from subsequent analyses. The number of alleles of the

17011 scorable loci ranged from six to 30 (Figure 1). Microsatellite genotype and collecting date for

171 each individual are available as supplementary information. For the Atlantic population, four loci

172 were not in HWE, and showed homozygote excess (Table 2). The estimated percentage of null

173 alleles ranged from $0 \%$ to $23 \%$. Allelic richness ranged from 3.65 to 20.7 , and genotyping error

174 rates ranged from 0 to $7 \%$ (Table 2 ).

175 All 11 loci amplified in some of the 23 Australian individuals, and had at least two alleles

176 (Table 3). No loci amplified in all individuals, and no individual failed to amplify all loci. For the

177 Pacific lineage of $C$. testudinaria, two loci deviated significantly from HWE expectations. The

178 locus Ctest7 showed heterozygote excess, even though not at a significant level $(\mathrm{p}$-value $=0.13)$.

179 Allelic richness of the Australian population was overall lower than in the Atlantic population.

180 The percentage of null alleles ranged from $0 \%$ to $33 \%$.

181

182

183 Discussion

184 The present study developed and characterized 11 microsatellite markers for the epizoic

185 barnacle Chelonibia testudinaria. Several loci are not in HWE, probably due to null alleles.

186 Their high allelic diversity and scoring consistency should nonetheless make them useful in

187 ecological and evolutionary studies. In addition, we provide the resources to evaluate thousands

188 of additional potentially amplifiable loci (PALs) for C. testudinaria.

189 Several loci were not in HWE, and displayed homozygote excess. Homozygote excess

190 can have several causes: selection on these loci, the presence of null alleles, inbreeding, 
191 population substructure or large variance in reproductive success. Inbreeding is unlikely because

192 most barnacles are obligate outcrossers and C. testudinaria has a widely-dispersing planktonic

193 larval phase. Selection cannot be excluded as an explanation, but selection on or near several

194 markers appears unlikely. Population substructure may be present, but if so, is neither host-

195 induced nor geographical. Large variance in reproductive success can cause homozygote excess

196 (Hedgecock, 1994), and has been invoked to explain homozygote excess in e.g. sea urchins

197 (Addison \& Hart, 2004). If variance in reproductive success is present, the effective population

198 size of C. testudinaria should be low (Hedgecock, 1994). We estimated a population mutation

199 rate (called theta, $\Theta$ ) of 10 for the Atlantic C. testudinaria population using Watterson's

200 estimator (Watterson, 1975) on published COI data, which suggests a large effective population

201 size (data not shown). These data do not support the variance-in-reproductive-success

202 hypothesis. The most likely cause for homozygote excess is the presence of null alleles. Null

203 alleles are ubiquitous in microsatellite loci, and are caused by mutations in the primer sequence,

204 leading to the dropout of alleles. While the true genotype is heterozygous, the observed genotype

205 is homozygous due to the non-amplification of one of the alleles. Null alleles become

206 increasingly prevalent with increasing effective population size (Chapuis and Estoup, 2007).

207 Chapuis and Estoup (2007) show that simulated null allele frequencies were larger than 0.2 for

208 all loci when $\Theta$ was one, the largest value they simulated. We estimated null allele frequencies

209 between zero and 0.3 for our microsatellite loci, well within the range of simulated data with

210 large effective population size. Thus the observed homozygote excess can be explained by the

211 presence of null alleles.

212 We were able to amplify all loci in both the Atlantic and Indo-West Pacific lineage,

213 which was somewhat surprising given large effective population size and significant between- 
214 lineage divergence. Both factors increase the chance for mutation accumulation in the primer

215 sequences between lineages. Further, results for the Indo-West Pacific lineage need to be

216 evaluated with caution because primers were designed from an individual of the Atlantic lineage.

217 As expected by the concept of ascertainment bias ( $\mathrm{Li}$ and Kimmel, 2013), the Atlantic lineage

218 had higher allelic richness than the Indo-West Pacific lineage. However, fewer loci violated the

219 expectations of HWE in the Indo-West Pacific lineage, likely an artifact of analyzing fewer

220 individuals, resulting in low power to detect significant deviations from HWE. Future studies

221 should increase sample sizes for both lineages to compare and contrast genotypic diversity.

222 Microsatellite markers have been developed successfully in a number of other barnacle

223 species (Dufresne et al. 1999; 2002; Dawson et al., 2010; Plough and Marko, 2014), providing

224 tools to address interesting ecological and evolutionary questions. We expect the loci developed

225 for C. testudinaria will be similarly useful.

\section{References}

229

230

231

232

233

234

235

236

Alberto, F. 2009. Msatallele_1.0: An r package to visualize the binning of microsatellite alleles. J. Hered. 100: 394-7.

Andrews, S. 2015. Fastqc a quality control tool for high throughput sequence data. http://www.bioinformatics.babraham.ac.uk/projects/fastqc/.

Aronesty, E. 2011. Fastq-mcf sequence quality filter, clipping and processor http://code.Google.com/p/ea-utils/wiki/fastqmcf.

Brookfield J. 1996. A simple new method for estimating null allele frequency from heterozygote deficiency. Molecular Ecology 5, 453-455. 
237 Castoe, T. A., Poole, A. W., de Koning, A. P. J., Jones, K. L., Tomback, D. F., Oyler-McCance,

238 S. J., Fike, J. A., Lance, S. L., Streicher, J. W., Smith, E. N. \& Pollock, D. D. 2012.

239 Rapid microsatellite identification from illumina paired-end genomic sequencing in two birds and a snake. PLOS ONE 7: e30953.

Chapuis, M.-P. \& Estoup, A. 2007. Microsatellite null alleles and estimation of population differentiation. Mol. Biol. Evol. 24: 621-631.

243

Cheang, C., Tsang, L., Chu, K., Cheng, I.-J. \& Chan, B. 2013. Host-specific phenotypic plasticity of the turtle barnacle Chelonibia testudinaria: A widespread generalist rather than a specialist. PLOS ONE 8: e57592.

Darwin, C. 1854. A monograph on the sub-class Cirripedia, with figures of all the species. The Balanidae, the Verrucidae, etc. Ray society, London.

Dawson MN, Grosberg RK, Yoel E S, Eric S. 2010. Population genetic analysis of a recent range expansion: mechanisms regulating the poleward range limit in the volcano barnacle Tetraclita rubescens. Molecular Ecology 19: 1585-1605.

Dufresne F, Bourget E, Bernatchez L. 2002. Differential patterns of spatial divergence in microsatellite and allozyme alleles: further evidence for locus-specific selection in the acorn barnacle, Semibalanus balanoides? Molecular Ecology 11:113-123.

Dufresne F, Parent M, Bernatchez L. 1999. Isolation and characterization of microsatellite markers in the acorn barnacle Semibalanus balanoides. Molecular Ecology 8:1558.

Gruvel, A. 1903. Cirrhipédes opercules nouveaux ou peu connus de la collection du muséum. Bull. Mus.: 23-25.

Hayashi, R. 2013. A checklist of turtle and whale barnacles (cirripedia: Thoracica: Coronuloidea). J. Mar. Biol. Assoc. U.K. 93: 143-182. 
260 Hedgecock, D. 1994. Does variance in reproductive success limit effective population sizes of 261 marine organisms. In: Genetics and the evolution of aquatic marine organisms,

262 (Beaumont, A. R., ed.). pp. 122-134. Chapman \& Hall, London.

263 Holm, S. 1979. A simple sequentially rejective multiple test procedure. Scandinavian Journal of 264 Statistics 6:65-70.

265 Jarne P, Lagoda PJ. 1996. Microsatellites, from molecules to populations and back. Trends in 266 Ecology \& Evolution 11, 424-429.

267 Kearse, M., Moir, R., Wilson, A., Stones-Havas, S., Cheung, M., Sturrock, S., Buxton, S., 268 Cooper, A., Markowitz, S., Duran, C., Thierer, T., Ashton, B., Mentjies, P. \& Bioinformatics 28: 1647-1649.

Li, B., Kimmel, M. 2013. Factors Influencing Ascertainment Bias of Microsatellite Allele Sizes: Impact on Estimates of Mutation Rates. Genetics 195: 563-572.

Linnaeus, C. 1758. Systema naturae per regna tria naturae, secundum classes, ordines, genera, species, cum characteribus, differentiis, synonymis, locis, 10 ed. Tomus I. L. Salvii, Stockholm, Sweden.

Paradis, E. 2010. Pegas: An R package for population genetics with an integrated-modular 278 approach. Bioinformatics: btp696.

Plough LV, Marko PB. 2013. Characterization of microsatellite loci and repeat density in the 280 gooseneck barnacle, Pollicipes elegans, using next generation sequencing. Journal of Heredity:est064.

282 Ranzani, A. C. 1818. Osservazioni su i balanidae parte iii. Opusculi Scientifici 2: 63-93. 
283 Rawson, P. D., Macnamee, R., Frick, M. G. \& Williams, K. L. 2003. Phylogeography of the 284 coronulid barnacle, Chelonibia testudinaria, from loggerhead sea turtles, caretta caretta. Mol. Ecol. 12: 2697-2706.

Regier J. C., Shultz, J. W., Zwick, A., Hussey, A., Ball, B., Wetzer, R., Martin, J. W., Cunningham, C. W. 2010. Arthropod relationships revealed by phylogenomic analysis of nuclear protein-coding sequences. Nature. 463: 1079-1083.

Rozen, S. \& Skaletsky, H. (2000) Primer3 on the www for general users and for biologist programmers. In: Bioinformatics methods and protocols: Methods in molecular biology, (Krawetz, S. \& Misener, S., eds.). pp. 365-386. Humana Press, Totowa, NJ.

Selkoe KA, Toonen RJ (2006) Microsatellites for ecologists: A practical guide to using and evaluating microsatellite markers. Ecology Letters 9, 615-629.

Schuelke, M. 2000. An economic method for the fluorescent labeling of PCR fragments. Nat

Vignal A, Milan D, SanCristobal M, Eggen A (2002) A review on SNP and other types of molecular markers and their use in animal genetics. Genetics Selection Evolution 34, 275306.

Walsh, P. S., Metzger, D. A. \& Higuchi, R. 1991. Chelex 100 as a medium for simple extraction of DNA for PCR-based typing from forensic material. BioTech. 10: 506-13.

Watterson, G. A. 1975. On the number of segregating sites in genetical models without recombination. Theoretical Population Biology 7: 256-276. 45-59. 
Figure 1 (on next page)

Allele frequencies of each microsatellite locus amplified from the Atlantic lineage of Chelonibia testudinaria

Each barplot represents a locus, each bar an allele, and the height of each bar indicates the frequency of each allele in in the data. Sample sizes are indicated in table 2. 
Figure 2 (on next page)

Allele frequencies of each microsatellite locus amplified from the Indo-West Pacific lineage of Chelonibia testudinaria

Each barplot represents a locus, each bar an allele, and the height of each bar indicates the frequency of each allele in in the data. Sample sizes are indicated in table 3. 
PEtest7

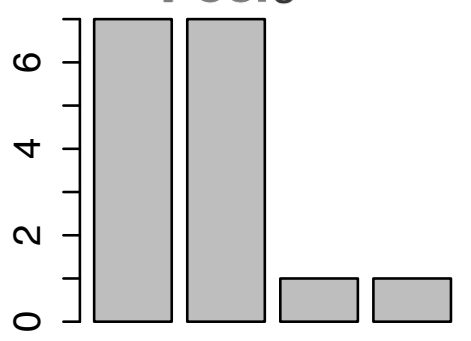

Ctest9

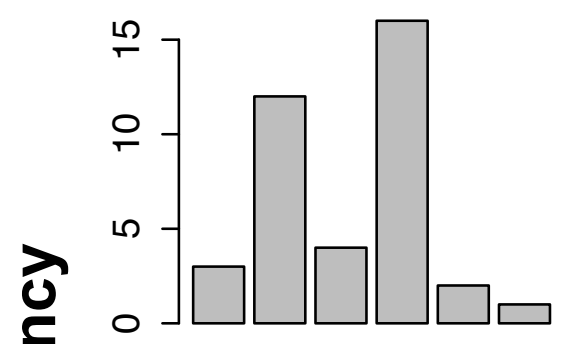

Ctest10

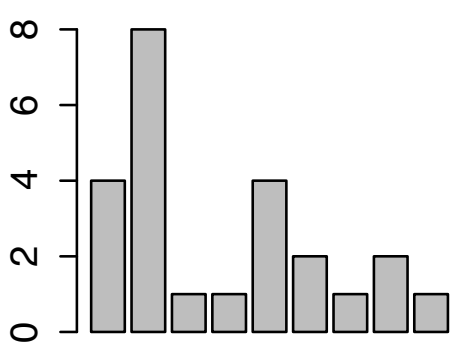

Ctest11

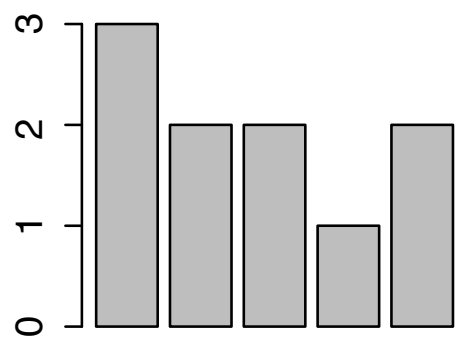

Ctest12 2 anuscript to be reviewed-est32

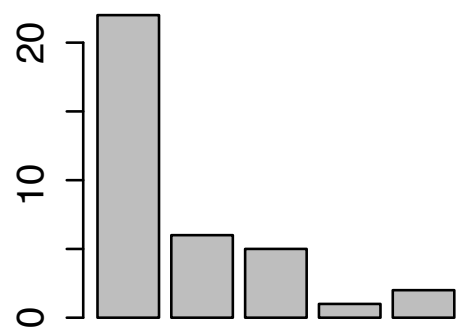

Ctest16

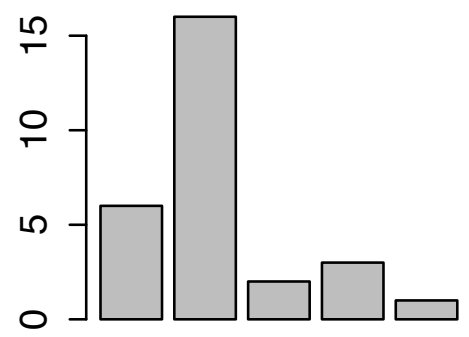

Ctest18

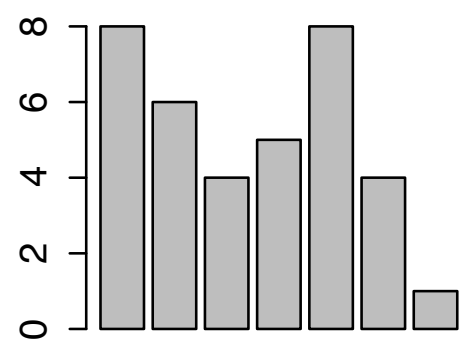

Ctest31

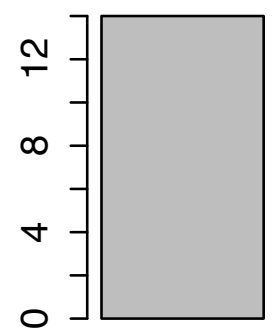

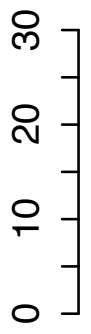

Ctest36

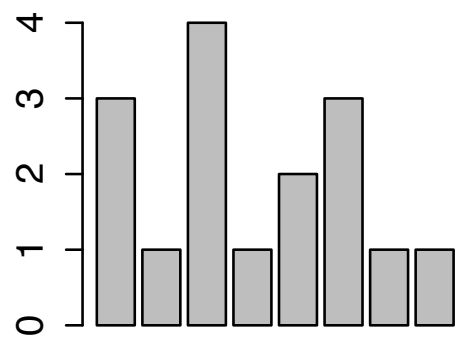

Ctest47

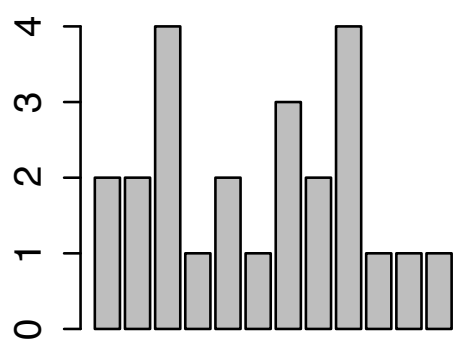




\section{Table $\mathbf{1}$ (on next page)}

Microsatellite loci amplification information

All loci were amplified at $55^{\circ} \mathrm{C}$ annealing temperature. "Dye" refers to the fluorescent color label for each forward primer. NED is yellow, 6-FAM is blue and HEX is green. Labeling forward primers with different colors allows multiplexing several primer sets in the same reaction. "Multiplex reaction" refers to the multiplexing PCR scheme, e.g. all loci with the same multiplex code were amplified in the same reaction. 
Table 1.

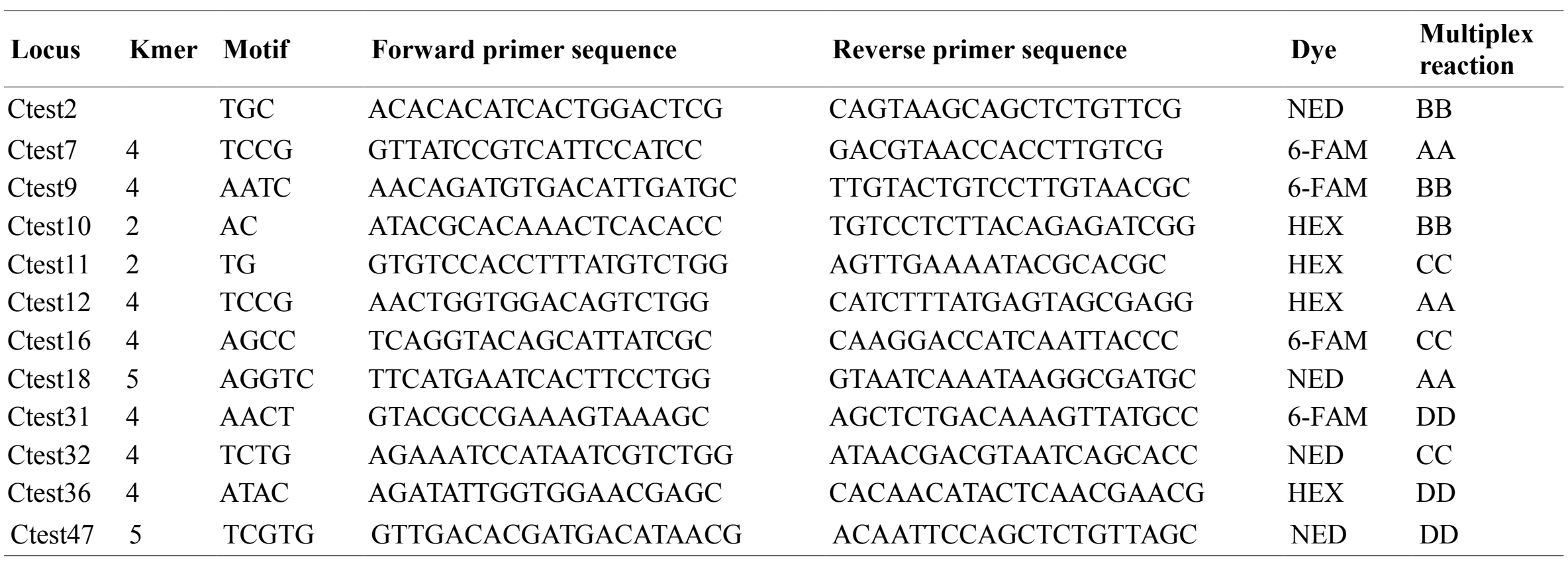




\section{Table 2 (on next page)}

Microsatellite loci characterization for Chelonibia testudinaria of the Atlantic lineage

Range refers to the smallest $(\mathrm{min})$ and largest $(\max )$ allele observed. Frequency of null alleles was estimated after Brookfield (1996). Significance values of HWE test were adjusted for multiple comparisons (Holm, 1979). Genotyping error rates were based on re-genotyping of all Atlantic individuals. Abbreviations: $\mathrm{n}=$ number of individuals; Obs het. $=$ observed heterozygosity; Exp het. = expected heterozygosity. 
Table 2.

\begin{tabular}{lllllllllll}
\hline Locus & $\mathbf{n}$ & $\begin{array}{l}\text { Range } \\
\text { min }\end{array}$ & $\begin{array}{l}\text { Range } \\
\text { max }\end{array}$ & $\begin{array}{l}\text { Number of } \\
\text { alleles }\end{array}$ & Obs het. & Exp het. & $\begin{array}{l}\text { HWE p- } \\
\text { value }\end{array}$ & $\begin{array}{l}\text { Allelic } \\
\text { richness }\end{array}$ & $\begin{array}{l}\text { Frequency null } \\
\text { alleles }\end{array}$ & $\begin{array}{l}\text { Genotyping } \\
\text { error rate }\end{array}$ \\
\hline Ctest7 & 34 & 206 & 314 & 18 & 0.56 & 0.82 & 0.09 & 16.11 & 0.14 & 0 \\
Ctest9 & 34 & 388 & 432 & 8 & 0.62 & 0.69 & $\mathbf{0 . 0 3}$ & 7.45 & 0.04 & 0.02 \\
Ctest10 & 34 & 264 & 278 & 8 & 0.65 & 0.77 & 0.13 & 7.45 & 0.07 & 0.03 \\
Ctest11 & 38 & 140 & 318 & 27 & 0.87 & 0.93 & 0.52 & 22.68 & 0.03 & 0.07 \\
Ctest12 & 34 & 388 & 476 & 23 & 0.74 & 0.92 & $\mathbf{0 . 0 1}$ & 20.26 & 0.1 \\
Ctest16 & 35 & 355 & 367 & 4 & 0.37 & 0.39 & 0.64 & 3.7 & 0.01 & 0.02 \\
Ctest18 & 33 & 450 & 485 & 7 & 0.61 & 0.78 & 0.18 & 6.98 & 0.1 \\
Ctest31 & 36 & 292 & 336 & 8 & 0.56 & 0.66 & $<\mathbf{0 . 0 0 0 1}$ & 6.54 & 0.07 \\
Ctest32 & 35 & 316 & 464 & 4 & 0.57 & 0.52 & 0.92 & 3.65 & 0 & 0.06 \\
Ctest36 & 33 & 336 & 524 & 23 & 0.46 & 0.89 & $<\mathbf{0 . 0 0 0 1}$ & 20.07 & 0.23 & 0.07 \\
Ctest47 & 37 & 255 & 385 & 10 & 0.62 & 0.66 & 0.78 & 8.36 & 0.02 & 0.03 \\
\hline
\end{tabular}




\section{Table 3 (on next page)}

Microsatellite loci characterization for Chelonibia testudinaria of the Indo-West Pacific lineage

Range refers to the smallest $(\mathrm{min})$ and largest $(\max )$ allele observed. Frequency of null alleles was estimated after Brookfield (1996). Significance values of HWE test were adjusted for multiple comparisons (Holm, 1979). Abbreviations: $\mathrm{n}=$ number of individuals; Obs het. $=$ observed heterozygosity; Exp het. = expected heterozygosity. 
Table 3.

\begin{tabular}{|c|c|c|c|c|c|c|c|c|c|}
\hline Locus & $\mathbf{n}$ & $\begin{array}{l}\text { Range } \\
\text { min }\end{array}$ & $\begin{array}{l}\text { Range } \\
\text { max }\end{array}$ & $\begin{array}{l}\text { Number of } \\
\text { alleles }\end{array}$ & Obs het. & Exp het. & $\begin{array}{l}\text { HWE p- } \\
\text { value }\end{array}$ & Allelic richness & $\begin{array}{l}\text { Frequency null } \\
\text { alleles }\end{array}$ \\
\hline Ctest7 & 8 & 186 & 314 & 4 & 1 & 0.61 & 0.13 & 3.07 & 0 \\
\hline Ctest9 & 19 & 408 & 456 & 6 & 0.74 & 0.7 & 1 & 3.95 & 0 \\
\hline Ctest10 & 12 & 260 & 344 & 9 & 0.5 & 0.81 & 0.08 & 5.48 & 0.17 \\
\hline Ctest11 & 5 & 136 & 236 & 5 & 0.2 & 0.78 & 0.11 & 4.63 & 0.33 \\
\hline Ctest12 & 18 & 354 & 374 & 5 & 0.33 & 0.58 & $<0.0001$ & 3.38 & 0.15 \\
\hline Ctest18 & 18 & 440 & 480 & 7 & 0.28 & 0.83 & $<0.0001$ & 5.22 & 0.3 \\
\hline Ctest31 & 8 & 224 & 304 & 2 & 0.25 & 0.22 & 1 & 1.8 & 0 \\
\hline Ctest32 & 19 & 308 & 316 & 3 & 0 & 0.28 & 0.08 & 2.13 & 0.22 \\
\hline Ctest36 & 8 & 348 & 468 & 8 & 0.5 & 0.84 & 0.1 & 5.74 & 0.18 \\
\hline Ctest 47 & 12 & 140 & 340 & 12 & 0.83 & 0.89 & 0.14 & 6.88 & 0.03 \\
\hline
\end{tabular}

